\title{
Self-Triggering under State-Independent
}

\section{Disturbances}

\author{
Xiaofeng Wang and Michael D. Lemmon
}

\begin{abstract}
This note studies self-triggering in sampled-data systems, where the next task release time and finishing time are predicted based on the sampled states. We propose a self-triggering scheme that ensures finite-gain $\mathcal{L}_{2}$ stability of the systems. This scheme relaxes the assumption in the previous work that the magnitude of the process noise is bounded by a linear function of the norm of the system state. We show that the sample periods generated by this scheme are always greater than a positive constant. We also provide state-based deadlines for delays and propose a way that may enlarge those deadlines without breaking $\mathcal{L}_{2}$ stability.
\end{abstract}

\section{INTRODUCTION}

Sampled-data systems are such systems that sample continuous signals and make control decisions based the sampled data. Traditional approaches to implement such systems are based on periodic task models, in which consecutive invocations of a task are released in a periodic manner [1], [2], [3], [4], [5], [6], [7]. Periodic task models, however, may be undesirable in many situations due to their conservativeness. Under periodic task models, the selection of sample periods is done before the system is deployed. One therefore has to ensure adequate behavior over a wide range of uncertainties. As a result, these selected periods may be shorter than necessary, which results in significant over-provisioning of the real-time system hardware. This over-provisioning may negatively impact the scheduling of other tasks on the same processing system. In these applications it may be better to consider alternatives to periodic task models

Both authors are with the department of electrical engineering, University of Notre Dame, Notre Dame, IN 46556; e-mail: xwang13,lemmon@nd.edu. The authors gratefully acknowledge the partial financial support of the National Science Foundation (grants NSF-CNS-0720457) 
that can more effectively balance the real-time system's computational cost against the control system's performance.

In recent years, sporadic task models have been considered for real-time control. A hardware realization of such models is called event-triggering. Under event-triggering the system states are sampled when some error signal exceeds a given threshold [8], [9], [10], [11], [12]. Eventtriggering has the ability to dynamically adjust the task periods to variations in the system state. This "on-line" property enables event-triggering to generate longer task periods than periodic task models [13]. One thing worth mentioning is that event-triggering requires hardware event detectors that may be implemented using application-specific integrated circuits (ASIC) or field-programmable gate array (FPGA) processors. In some applications, however, it may be unreasonable or impractical to retrofit an existing system with such "event detectors". In these cases, a software approach such as the self-triggered scheme may be more appropriate.

Under self-triggering the next task release time and finishing time are predicted by the processing computer based on the sampled data. A self-triggered task model was introduced by Velasco et al. [14] in which a heuristic rule was used to adjust task periods. Further work was done by Lemmon et al. [15] which chose task periods based on a Lyapunov-based technique. But the authors did not provide analytic bounds for task periods. Most recently, Wang et al. [13] provided the first rigorous examination of what might be required to implement self-triggered feedback control systems for $\mathcal{L}_{2}$ stability. A space-time scaling law for the execution times of control tasks was derived in [16] for asymptotic stability of homogeneous systems.

A critical assumption in [13] is that the magnitude of the process noise is bounded by a linear function of the norm of the system state. It means that the disturbance should vanish as the state is close to the equilibrium. Such disturbances may arise in uncertain systems when there are unmodeled dynamics caused by fluctuations in plant parameters. In practice, however, the disturbances usually do not depend on the state. With those "independent" disturbances, the self-triggering scheme in [13] cannot theoretically guarantee $\mathcal{L}_{2}$ stability of the sampled-data system any more. It is, therefore, important to relax this assumption so that the self-triggering scheme can apply to a wider class of systems.

This note extends the work in [13]. We present a novel self-triggering scheme that ensures finite-gain $\mathcal{L}_{2}$ stability of the resulting self-triggered feedback systems. This scheme pertains to linear time-invariant systems. The task release time and finishing time are predicted as functions 
of previously sampled states. It relaxes the assumption in [13] that the magnitude of the process noise is bounded by a linear function of the norm of the system state. We show that the sample periods generated by this scheme are always greater than a positive constant. We also provide state-based deadlines for delays and propose a way that may enlarge predicted deadlines without breaking $\mathcal{L}_{2}$ stability of the system. Simulations show that the sample periods generated by our scheme are longer than those generated by the scheme in [13].

This note is organized as follows. Section II introduces the system model. Section III presents self-triggering schemes for sampled-data systems. Simulation results are presented in section IV and conclusions are stated in section $\mathrm{V}$.

\section{SYSTEM MODEL}

We consider a sampled-data implementation of linear time-invariant closed-loop systems. In such systems, the plant's control, $u$, is computed by a computer task. This task is characterized by two monotone increasing sequences of time instants; the release time sequence $\left\{r_{k}\right\}_{k=0}^{\infty}$ and the finishing time sequence $\left\{f_{k}\right\}_{k=0}^{\infty}$. The time $r_{k}$ denotes the time when the $k$ th invocation of a control task (also called a job) is released for execution on the computer's central processing unit (CPU). The time $f_{k}$ denotes the time when then $k$ th job has finished executing. Notice that one job includes sampling the state, computing the control input, and feeding the input back to the plant. The control $u$ is computed based on the last sampled state. The control signal used by the plant is held constant by a zero-order hold $(\mathrm{ZOH})$ until the next finishing time $f_{k+1}$. This means that the sampled-data system under study satisfies,

$$
\begin{aligned}
& \dot{x}_{t}=A x_{t}+B_{1} u_{t}+B_{2} w_{t} \\
& u_{t}=-B_{1}^{T} P x_{r_{k}}
\end{aligned}
$$

for $t \in\left[f_{k}, f_{k+1}\right)$ and all $k \in \mathbb{N}$. In equation (1), $x:[0, \infty) \rightarrow \mathbb{R}^{n}$ is the state trajectory, $u:[0, \infty) \rightarrow \mathbb{R}^{m}$ is a control input, $w:[0, \infty) \rightarrow \mathbb{R}^{l}$ is an exogenous disturbance function in $\mathcal{L}_{2}$ space, and $P \in \mathbb{R}^{n \times n}$ is a symmetric positive semi-definite matrix satisfying the $\mathcal{H}_{\infty}$ algebraic Riccati equation (ARE),

$$
0=P A+A^{T} P-P B_{1} B_{1}^{T} P+I+\frac{1}{\gamma^{2}} P B_{2} B_{2}^{T} P
$$

for some real constant $\gamma>0$. For notational convenience, let $A_{\mathrm{cl}}=A-B_{1} B_{1}^{T} P, e_{t}^{k}=x_{t}-x_{r_{k}}$ denote the measurement error, $T_{k}=r_{k+1}-r_{k}$ denote the $k$ th inter-release time (known as 
"sample period") and $D_{k}=f_{k}-r_{k}$ denote the time interval between the $k$ th job's release and finishing time (known as "delay").

Definition 2.1: The system (1) is said to be finite-gain $\mathcal{L}_{2}$ stable from $w$ to $x$ with an induced gain less than $\gamma$ if there exist non-negative constants $\gamma$ and $\delta$ such that

$$
\left(\int_{0}^{\infty}\left\|x_{t}\right\|_{2}^{2} d t\right)^{\frac{1}{2}} \leq \gamma\left(\int_{0}^{\infty}\left\|w_{t}\right\|_{2}^{2} d t\right)^{\frac{1}{2}}+\delta
$$

for any $w$ satisfying $\left(\int_{0}^{\infty}\left\|w_{t}\right\|_{2}^{2} d t\right)^{\frac{1}{2}}<\infty$.

Objective: Design a self-triggering scheme to determine $r_{k}$ and $f_{k}$ such that finite-gain $\mathcal{L}_{2}$ stability can be preserved for $w$ to $x$.

\section{Self-triggered Feedback Systems}

This section proposes a self-triggering scheme for sampled-data systems. This scheme ensures finite-gain $\mathcal{L}_{2}$ stability of the resulting sampled-data systems. The idea is that we first seek realtime constraints on the time instants $r_{k}$ and $f_{k}$ such that $\mathcal{L}_{2}$ stability of the sampled-data system can be guaranteed; then we derive a self-triggering scheme that ensures the satisfaction of these constraints.

Before we show the desired real-time constraints, we need a lemma to help the proof, which provide an upper bound for the derivative of the storage function. To make the note easy to read, we put all of the proofs in the appendix.

Lemma 3.1: Consider the sampled-data system in equation (1). Let $V: \mathbb{R}^{n} \rightarrow \mathbb{R}^{+}$be a positive semi-definite function defined by $V(x)=x^{T} P x$ with the matrix $P$ given in equation (2). For any real constant $\beta \in(0,1]$, the directional derivative of $V$ satisfies

$$
\dot{V} \leq-\beta^{2}\left\|x_{t}\right\|_{2}^{2}+\gamma^{2}\left\|w_{t}\right\|_{2}^{2}+\left(e_{t}^{k}\right)^{T} M e_{t}^{k}-x_{r_{k}}^{T} N x_{r_{k}}
$$

holds for all $t \in\left[f_{k}, f_{k+1}\right)$ and all $k \in \mathbb{N}$, where $M, N$ satisfy

$$
\begin{aligned}
M & =\left(1-\beta^{2}\right) I+P B_{1} B_{1}^{T} P \\
N & =\frac{1}{2}\left(1-\beta^{2}\right) I+P B_{1} B_{1}^{T} P,
\end{aligned}
$$

respectively.

It is easy to see that if we can enforce

$$
\left(e_{t}^{k}\right)^{T} M e_{t}^{k} \leq \rho\left(x_{r_{k}}\right)^{2}
$$


for all $t \in\left[f_{k}, f_{k+1}\right)$, then the inequality in equation (4) implies

$$
\dot{V} \leq-\beta^{2}\left\|x_{t}\right\|_{2}^{2}+\gamma^{2}\left\|w_{t}\right\|_{2}^{2}
$$

which means $\mathcal{L}_{2}$ stability. That is the main idea in [13]. It is, however, difficult to predict the future time when we expect inequality (7) to be violated due to the uncertainty $w_{t}$ involved in $e_{t}^{k}$. To solve this issue, an assumption was made in [13] that $\left\|w_{t}\right\|_{2} \leq W\left\|x_{t}\right\|_{2}$ must hold for some positive constant $W \in \mathbb{R}$. In this way, uncertainty can be reduced. This assumption may be justified if the noise term is generated by state-dependent modeling uncertainty, but in general if the disturbance is independent of the process model, this assumption will be overly restrictive.

We are interested in relaxing this assumption so that $w_{t}$ can be any signal in $\mathcal{L}_{2}$ space. We are able to do this by splitting the effect that the sampled state $x_{r_{k}}$ and the noise $w_{t}$ have on the error $e_{t}^{k}$ for $t \in\left[f_{k}, f_{k+1}\right)$. In other words, we bound $\left\|\sqrt{M} e_{t}^{k}\right\|_{2}$ by a functional of $w$ plus another term that is only a function of the previously sampled states $x_{r_{k}}$ and $x_{r_{k-1}}$. Lemma 3.2 provides such a bound on $\left\|\sqrt{M} e_{t}^{k}\right\|_{2}$ over $\left[f_{k}, f_{k+1}\right)$.

For the notation convenience, we define $z: \mathbb{R} \times \mathbb{N} \rightarrow \mathbb{R}^{n}, \rho: \mathbb{R}^{n} \rightarrow \mathbb{R}, \mu_{0}: \mathbb{R}^{n} \rightarrow \mathbb{R}$, $\mu_{1}: \mathbb{R}^{n} \times \mathbb{R}^{n} \rightarrow \mathbb{R}$, and $\alpha \in \mathbb{R}$ as

$$
\begin{aligned}
& z_{t}^{k}=\sqrt{M} e_{t}^{k} \\
& \rho(x)=\sqrt{x^{T} N x} \\
& \mu_{0}\left(x_{r_{k}}\right)=\left\|\sqrt{M} A_{\mathrm{cl}} x_{r_{k}}\right\|_{2} \\
& \mu_{1}\left(x_{r_{k}}, x_{r_{k-1}}\right)=\left\|\sqrt{M}\left(A x_{r_{k}}-B_{1} B_{1}^{T} P x_{r_{k-1}}\right)\right\|_{2} \\
& \alpha=\left\|\sqrt{M} A \sqrt{M}^{-1}\right\|,
\end{aligned}
$$

respectively.

Lemma 3.2: Consider the sampled-data system in equation (1). Let $\beta$ be any positive constant in the interval $(0,1]$ such that the matrix $M$ defined in equation (5) has full rank. If $r_{k} \leq f_{k} \leq$ $f_{k+1}$ holds for some $k \in \mathbb{N}$, then the following inequality must hold:

$$
\begin{aligned}
\left\|\sqrt{M} e_{t}^{k}\right\|_{2} \leq & e^{\alpha\left(t-f_{k}\right)} \frac{\mu_{1}\left(x_{r_{k}}, x_{r_{k-1}}\right)}{\alpha}\left(e^{\alpha D_{k}}-1\right)+\frac{\mu_{0}\left(x_{r_{k}}\right)}{\alpha}\left(e^{\alpha\left(t-f_{k}\right)}-1\right) \\
& +e^{\alpha\left(t-f_{k}\right)} J\left[w ; r_{k}, f_{k}\right]+J\left[w ; f_{k}, t\right]
\end{aligned}
$$

holds for all $t \in\left[f_{k}, f_{k+1}\right)$, where

$$
J[w ; a, b]=\int_{a}^{b} e^{\alpha(b-s)}\left\|\sqrt{M} B_{2}\right\|\left\|w_{s}\right\|_{2} d s .
$$


With the preceding lemmas, we are able to present the theorem that gives inequality constraints on $r_{k}$ and $f_{k}$.

Theorem 3.3: Consider the sampled-data system in equation (1). Let $\beta$ be any positive constant in the interval $(0,1]$ such that the matrix $M$ defined in equation (5) has full rank. Given three positive constants $\epsilon \in[0,1], \tau_{1}, \tau_{2} \in \mathbb{R}^{+}$and a positive sequence $\left\{\delta_{k}\right\}_{k=0}^{\infty}$ satisfying $\sum_{k=0}^{\infty} \delta_{k} \leq$ $\infty$, if for any $k \in \mathbb{N}$, the following inequalities

$$
\begin{aligned}
& r_{k} \leq f_{k} \leq r_{k+1} \\
& r_{k+1}-f_{k} \leq \tau_{1} \\
& f_{k}-r_{k} \leq \tau_{2} \\
& \sigma\left(x_{r_{k}}, x_{r_{k-1}}, f_{k+1}, f_{k}, D_{k}\right) \leq\left(x_{r_{k}}^{T} N x_{r_{k}}+\delta_{k}\right)\left(f_{k+1}-f_{k}\right)
\end{aligned}
$$

hold, where

$$
\begin{aligned}
\sigma\left(x_{r_{k}}, x_{r_{k-1}}, f_{k+1}, f_{k}, D_{k}\right)= & \frac{2 \mu_{1}^{2}\left(x_{r_{k}}, x_{r_{k-1}}\right)}{\alpha^{3}}\left(e^{\alpha D_{k}}-1\right)^{2}\left(e^{2 \alpha\left(f_{k+1}-f_{k}\right)}-1\right)+\frac{4 \mu_{0}^{2}\left(x_{r_{k}}\right)}{\alpha^{2}}\left(f_{k+1}-f_{k}\right) \\
& +\frac{2 \mu_{0}^{2}\left(x_{r_{k}}\right)}{\alpha^{3}}\left(e^{2 \alpha\left(f_{k+1}-f_{k}\right)}-1\right)-\frac{8 \mu_{0}^{2}\left(x_{r_{k}}\right)}{\alpha^{3}}\left(e^{\alpha\left(f_{k+1}-f_{k}\right)}-1\right),
\end{aligned}
$$

then the sampled-data system is finite-gain $\mathcal{L}_{2}$ stable from $w$ to $x$ with an induced gain less than a positive constant $\eta$, where

$$
\eta=\frac{\sqrt{\gamma^{2} \alpha^{2}+\left\|\sqrt{M} B_{2}\right\|^{2}\left(\left(e^{2 \alpha\left(\tau_{1}+\tau_{2}\right)}-1\right)\left(e^{2 \alpha \tau_{2}}-1\right)+4\left(e^{\alpha\left(\tau_{1}+\tau_{2}\right)}-1\right)^{2}\right)}}{\alpha \beta} .
$$

Remark 3.4: The introduction of $\tau_{1}, \tau_{2}$ is the safety requirement of systems. It requires the system updates at least every $\tau_{1}+\tau_{2}$ unit-time so that some accidents can be detected. Notice that $\tau_{1}$ and $\tau_{2}$ also affect the induced gain.

Remark 3.5: The introduction of $\delta_{k}$ can enlarge the threshold on $\sigma$, as we can see in equation (18). With a large threshold, we have more flexibility to choose the sampling periods and deadlines. In the following discussion, $\delta_{k}$ is chosen in a way that can enlarge the predicted deadlines for delays.

Theorem 3.3 provides constraints on task release and finishing time instants in equations (15) (18). These constraints are algebraic inequalities. Given $x_{r_{k}}, x_{r_{k-1}}$, and $D_{k}$, let $\xi\left(x_{r_{k}}, x_{r_{k-1}}, D_{k}\right)$ be an upper bound on $f_{k+1}-f_{k}$ such that the inequality in equation (18) holds. Then with some $\epsilon \in[0,1]$, we may have a self-triggering scheme where the next release time $r_{k+1}$ is

$$
r_{k+1}=f_{k}+\min \left\{\tau_{1}, \epsilon \xi\left(x_{r_{k}}, x_{r_{k-1}}, D_{k}\right)\right\}
$$


and the $k+1$ st delay satisfies

$$
D_{k+1} \leq \min \left\{\tau_{2},(1-\epsilon) \xi\left(x_{r_{k}}, x_{r_{k-1}}, D_{k}\right)\right\}
$$

If we can guarantee $\xi\left(x_{r_{k}}, x_{r_{k-1}}, D_{k}\right) \geq 0$ for all $k \in \mathbb{N}$, then the scheme can ensure the satisfaction of equations (15) - (18) and therefore the sampled-data system is $\mathcal{L}_{2}$ stable.

According to the above discussion, it is important to ensure $\xi\left(x_{r_{k}}, x_{r_{k-1}}, D_{k}\right) \geq 0$. Notice that when $D_{k}=0, \xi\left(x_{r_{k}}, x_{r_{k-1}}, D_{k}\right) \geq 0$ always holds. When $D_{k}>0$, we need more constraints on $D_{k}$ besides equation (22) such that $\xi\left(x_{r_{k}}, x_{r_{k-1}}, D_{k}\right) \geq 0$ holds. Another challenge lies in the computation of $\xi\left(x_{r_{k}}, x_{r_{k-1}}, D_{k}\right)$. It is hard to obtain the explicit form of $\xi$ in terms of $x_{r_{k}}, x_{r_{k-1}}$, and $D_{k}$. As a result, the inequalities must be solved online. It requires a lot of computational resource, which is unexpected in real-time control. An alternative way is to obtain a more conservative self-triggering scheme that ensures the satisfaction of equation (18), but uses less computational resource. The following theorem addresses these issues.

Theorem 3.6: Consider the sampled-data system in equation (1). Let $\beta$ be any positive constant in the interval $(0,1]$ such that the matrix $M$ defined in equation (5) has full rank. Given positive constants $\epsilon \in[0,1], \tau_{1}, \tau_{2} \in \mathbb{R}^{+}$and a positive sequence $\left\{\delta_{k}\right\}_{k=0}^{\infty}$ satisfying $\sum_{k=0}^{\infty} \delta_{k} \leq \infty$, if

- the initial condition is $r_{0}=f_{0}=0$,

- the $k+1$ st task release time $r_{k+1}$ satisfies

$$
r_{k+1}=f_{k}+\min \left\{\tau_{1}, \epsilon L_{2}\left(x_{r_{k}}\right)\right\}
$$

for all $k \in \mathbb{Z}^{+}$, where $L_{2}: \mathbb{R}^{n} \rightarrow \mathbb{R}$ is defined by

$$
L_{2}\left(x_{r_{k}}\right)=\left\{\begin{array}{cc}
\frac{1}{\alpha} \ln \left(1+\frac{\alpha \rho\left(x_{r_{k}}\right)}{\sqrt{8} \mu_{0}\left(x_{r_{k}}\right)}\right) & x_{r_{k}} \neq 0 \\
\infty & x_{r_{k}}=0
\end{array}\right.
$$

- the $k+1$ st task finishing time $f_{k+1}$ satisfies

$$
D_{k+1}=f_{k+1}-r_{k+1} \leq \min \left\{\tau_{2},(1-\epsilon) L_{2}\left(x_{r_{k}}\right), L_{3}\left(x_{r_{k+1}}, x_{r_{k}} ; \delta_{k+1}\right)\right\}
$$

where $L_{3}: \mathbb{R}^{n} \times \mathbb{R}^{n} \times \mathbb{R} \rightarrow \mathbb{R}$ is defined by

$$
L_{3}\left(x_{r_{k+1}}, x_{r_{k}} ; \delta_{k+1}\right)=\frac{1}{\alpha} \ln \left(1+\frac{\alpha \sqrt{\rho^{2}\left(x_{r_{k+1}}\right)+2 \delta_{k+1}}}{\sqrt{8} e^{\alpha \min \left\{\tau_{1}+\tau_{2}, L_{2}\left(x_{r_{k}}\right)\right\}_{\mu_{1}\left(x_{r_{k+1}}, x_{r_{k}}\right)}}}\right),
$$

then the sampled-data system is finite-gain $\mathcal{L}_{2}$ stable from $w$ to $x$ with an induced gain less than a positive constant $\eta$, where $\eta$ is defined in equation (20). 
Remark 3.7: In the self-triggering scheme defined by equations (23)-(26), the $k+1$ st task release time is determined when $t=f_{k}$. The deadline for the $k+1$ st task delay is determined when $t=r_{k+1} . \tau_{1}$ and $\tau_{2}$ are used to bound the time intervals $\left[f_{k}, r_{k+1}\right)$ and $\left[r_{k+1}, f_{k+1}\right)$, respectively, for the consideration of the system security.

Remark 3.8: The prediction of the deadline may take some computational resource. One may wonder whether it is better to use this resource to compute control input directly. This might be true if the delays are only caused by the computation of control inputs. In many situations, however, the computation is not the main reason to cause delays. In those cases, it is still worthy of using some computational resource to predict deadlines. This is particularly true in networked systems, where communication resource is much more expensive than computational resource.

Remark 3.9: By the definition of $L_{2}$, we know that $L_{2}\left(x_{r_{k}}\right)$ is always greater than a positive constant. Since $T_{k} \geq L_{2}\left(x_{r_{k}}\right)$, it implies the sample periods generated by this self-triggering scheme are always greater than a positive constant. This is important to establish because it avoids continuously sampling.

Remark 3.10: Equation (25) implies $D_{k} \leq L_{3}\left(x_{r_{k}}, x_{r_{k-1}} ; \delta_{k}\right)$. If we re-visit the self-triggering scheme in equations (21) and (22), this inequality actually ensures $\xi\left(x_{r_{k}}, x_{r_{k-1}}, D_{k}\right) \geq 0$.

Remark 3.11: The introduction of $\delta_{k}$ can increase the value of $L_{3}\left(x_{r_{k}}, x_{r_{k-1}} ; \delta_{k}\right)$. This suggests that by selecting large $\delta_{k}$, we can enlarge the predicted deadlines, provided that the deadlines are dominated by $L_{3}$. It would be an interesting research topic in the future on the selection of $\delta_{k}$ to ensure the deadlines are greater than a positive constant.

\section{Simulations}

In this section, we used the inverted pendulum problem in [13] to demonstrate the proposed self-triggered scheme. The plant's linearized state equations were

$$
\dot{x}_{t}=\left[\begin{array}{cccc}
0 & 1 & 0 & 0 \\
0 & 0 & -m_{1} g / m_{2} & 0 \\
0 & 0 & 0 & 1 \\
0 & 0 & g / \ell & 0
\end{array}\right] x_{t}+\left[\begin{array}{c}
0 \\
1 / m_{2} \\
0 \\
-1 /\left(m_{2} \ell\right)
\end{array}\right] u_{t}+\left[\begin{array}{l}
1 \\
1 \\
1 \\
1
\end{array}\right] w_{t}
$$

where $m_{1}$ was the mass of the pendulum bob, $m_{2}$ was the cart mass, $\ell$ was the length of the pendulum arm, and $g$ was gravitational acceleration. For these simulations, we let $m_{1}=1$, $m_{2}=10, \ell=3$, and $g=10$. The system state was the vector $x=\left[\begin{array}{llll}y & \dot{y} & \theta & \dot{\theta}\end{array}\right]^{T}$ where $y$ 
was the cart's position and $\theta$ was the pendulum bob's angle with respect to the vertical. The system's initial state was the vector $x_{0}=\left[\begin{array}{llll}0.98 & 0 & 0.2 & 0\end{array}\right]^{T}$. The controller was $u=K x$, where $K=\left[\begin{array}{llll}2 & 12 & 378 & 210\end{array}\right] \cdot \gamma$ was set to be 200 .

We compared the sampling period generated by three different self-triggering schemes: the scheme defined by equations (23)-(26), the scheme defined by equations (21)-(22), and the scheme proposed in [13]. In the simulations, we assumed that the delays are zero. For the first two schemes, we set $\tau_{1}=0.3, \tau_{2}=0, \beta=0.5, \delta_{k}=0$ and $\epsilon=1$. The induced gains in the first two systems, therefore, are less than 4926. according to equation (20).

Recall that the self-triggering scheme in [13] requires $\left\|w_{t}\right\|_{2} \leq W\left\|x_{t}\right\|_{2}$ holds for some $W>0$ and the $k+1$ st task release, $r_{k+1}$, is triggered in the following way:

$$
r_{k+1}=r_{k}+\frac{1}{\hat{\alpha}} \ln \left(1+\frac{\hat{\alpha}\left\|\sqrt{\hat{N}} x_{r_{k}}\right\|_{2}}{\left\|\sqrt{\hat{M}} A_{c l} x_{r_{k}}\right\|_{2}}\right),
$$

where

$$
\begin{aligned}
\hat{M} & =\left(1-\hat{\beta}^{2}\right) I+P B_{1} B_{1}^{T} P, \\
\hat{N} & =\frac{1}{2}\left(1-\hat{\beta}^{2}\right) I+P B_{1} B_{1}^{T} P, \\
\bar{\alpha} & =\left\|\sqrt{M} A \sqrt{M}^{-1}\right\|+W\left\|\sqrt{M} B_{2}\right\|\left\|\sqrt{M}^{-1}\right\| .
\end{aligned}
$$

The induced gain of the resulting self-triggered system is less than $\frac{\gamma}{\hat{\beta}}$. To make it a fair comparison, we set $\hat{\beta}=0.0406$ such that the theoretical bounds on the induced gains in the three self-triggered feedback systems are the same.

We first ran the self-triggered systems in [13] with a disturbance satisfying $\left\|w_{t}\right\|_{2} \leq 0.01\left\|x_{t}\right\|_{2}$ $(W=0.01)$. Then the same disturbance was added into the other two self-triggered systems. Figure 1 shows the periods generated by different schemes. It is obvious that the periods generated by our self-triggering schemes, in general, are much longer than those by the scheme in [13]. It suggests that our schemes are not conservative. Also notice that the scheme by equation (21)(22) has the longer periods than the scheme by (23)- (26). This is because the periods in the latter scheme are basically a conservative estimate of the periods in the former scheme.

We then examined the effect of $\delta_{k}$ on the deadlines in the self-triggered feedback scheme in equations $(23)-(26)$. The parameters are $\tau_{1}=0.3, \tau_{2}=0.2, \beta=0.5$, and $\epsilon=0.5$. We assumed that the delays are equal to the predicted deadlines and $w_{t}=0$. Two different cases 


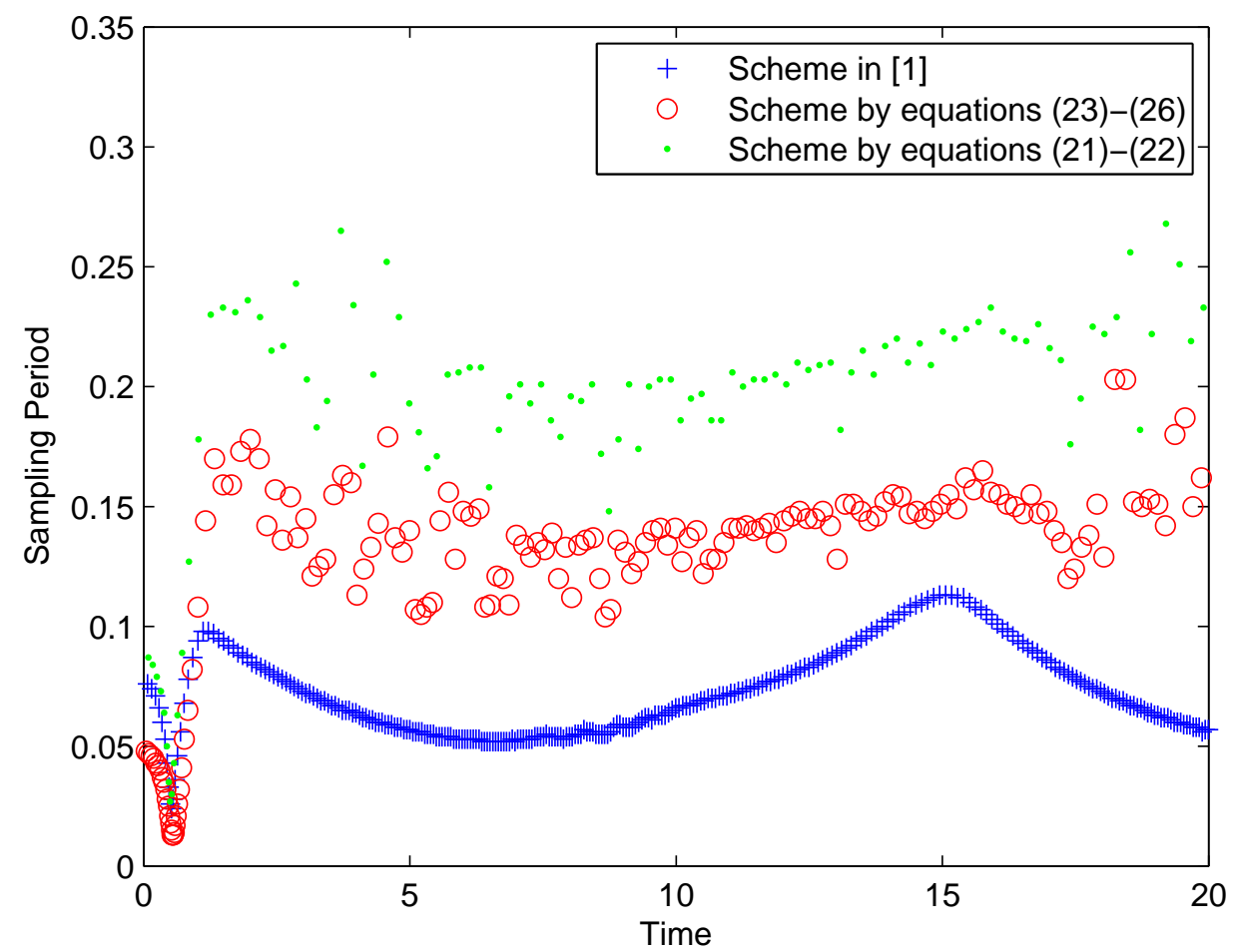

Fig. 1. A comparison of the sampling period generated by the scheme in equations (23)-(26) (circle), the scheme in equations (21)-(22) (dot), and the scheme in [13] (cross)

were considered: $\delta_{k}=0$ and $\delta_{k}=\frac{10^{5}}{k^{2}}$. In the simulation, both self-triggered feedback systems approached the equilibrium after the systems ran for 30 seconds. Figure 2 plots the predicted deadlines in the systems with $\delta_{k}=\frac{10^{5}}{k^{2}}$ (diamond) and $\delta_{k}=0$ (dot). It is obvious that the predicted deadlines with $\delta_{k}=\frac{10^{5}}{k^{2}}$ are much longer than those in the system with $\delta_{k}=0$. It suggests that appropriate selection of $\delta_{k}$ can result in longer deadlines, which might be useful in dealing with very short deadlines. Another interesting observation is that when $k$ becomes large and therefore $\delta_{k}$ becomes small, the level of the increase in deadlines remains the same. It suggests that our method is not only a solution of temporarily increasing deadlines. It may work over the entire time zone. How to efficiently select $\delta_{k}$ would be an interesting research topic in the future. 


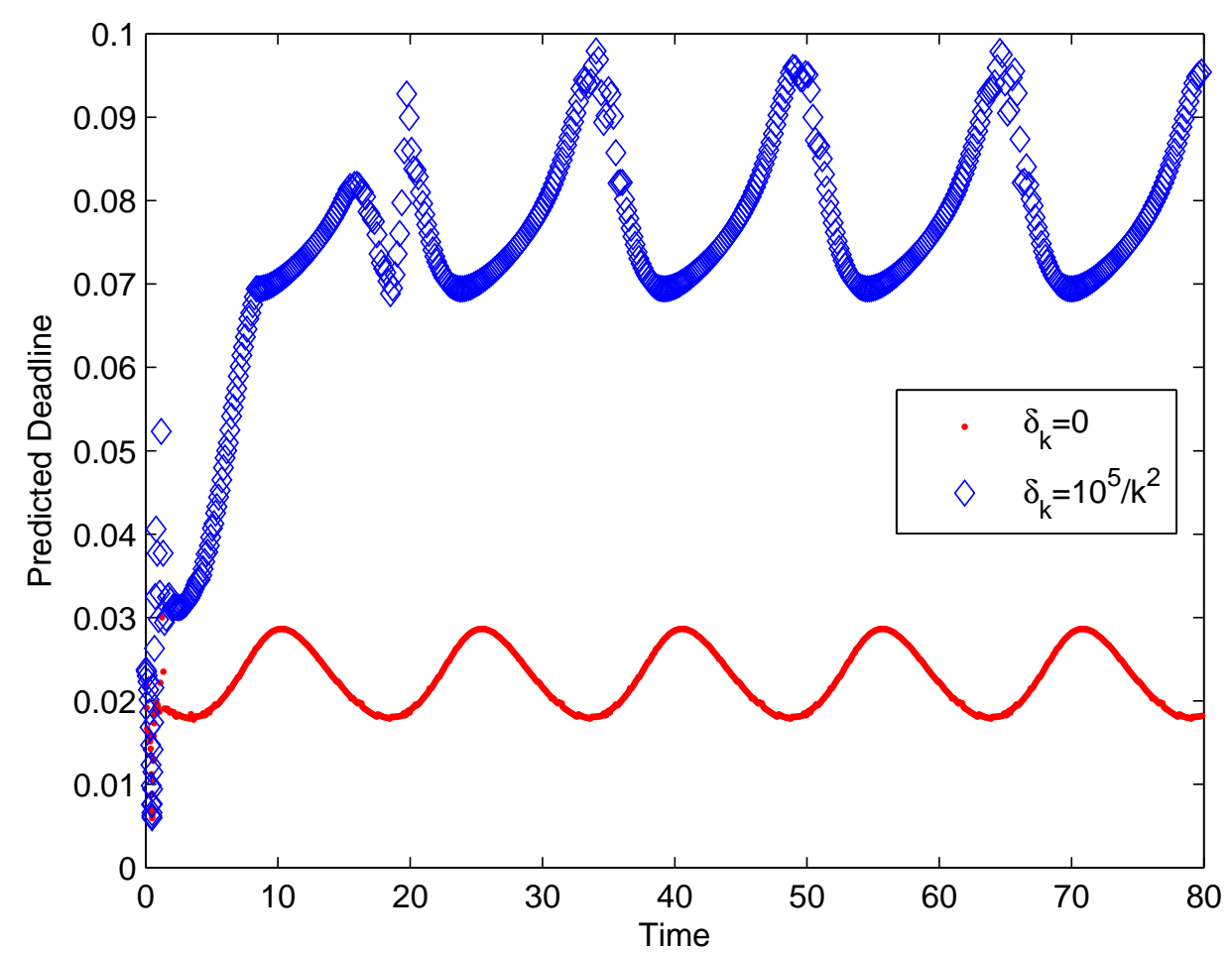

Fig. 2. A comparison between the predicted deadlines generated by the systems in equations (23)-(26) with $\delta_{k}=\frac{10^{5}}{k^{2}}$ (circle) and $\delta_{k}=0$ (dot)

\section{CONCLuSions}

This note proposes a self-triggering scheme that ensures finite-gain $\mathcal{L}_{2}$ stability of the resulting self-triggered feedback systems. This scheme relaxes the assumptions in [13] that the magnitude of the process noise is bounded by a linear function of the norm of the system state. We show that the sample periods generated by this scheme are always greater than a positive constant. We also provide dynamic deadlines for delays and propose a way that may enlarge predicted deadlines without breaking $\mathcal{L}_{2}$ stability. 


\section{APPENDIX}

Proof: [Proof of Lemma 3.1] Let $Q=P B_{1} B_{1}^{T} P$. Consider the directional derivative of $V$ at $t \in\left[f_{k}, f_{k+1}\right)$ :

$$
\begin{aligned}
\dot{V} & =\frac{\partial V}{\partial x}\left(A x_{t}-B_{1} B_{1}^{T} P x_{r_{k}}+B_{2} w_{t}\right) \\
& =-x_{t}^{T}(I-Q) x_{t}-\left\|\gamma w_{t}-\frac{1}{\gamma} B_{2}^{T} P x_{t}\right\|_{2}^{2}+\gamma^{2}\left\|w_{t}\right\|_{2}^{2}-2 x_{t}^{T} Q x_{r_{k}} \\
& \leq-x_{t}^{T}(I-Q) x_{t}+\gamma^{2}\left\|w_{t}\right\|_{2}^{2}-2 x_{t}^{T} Q x_{r_{k}} .
\end{aligned}
$$

Insert $x_{t}=e_{t}^{k}+x_{r_{k}}$ into the above equation to obtain

$$
\begin{aligned}
\dot{V} & \leq-\left\|x_{t}\right\|_{2}^{2}+\left(e_{t}^{k}+x_{r_{k}}\right)^{T} Q\left(e_{t}^{k}+x_{r_{k}}\right)-2\left(e_{t}^{k}+x_{r_{k}}\right)^{T} Q x_{r_{k}}+\gamma^{2}\left\|w_{t}\right\|_{2}^{2} \\
& =-\beta^{2}\left\|x_{t}\right\|_{2}^{2}-\left(1-\beta^{2}\right)\left\|x_{t}\right\|_{2}^{2}+\left(e_{t}^{k}\right)^{T} Q e_{t}^{k}-x_{r_{k}}^{T} Q x_{r_{k}}+\gamma^{2}\left\|w_{t}\right\|_{2}^{2} .
\end{aligned}
$$

Notice that

$$
\begin{aligned}
\left\|x_{t}\right\|_{2}^{2} & =\left\|e_{t}^{k}+x_{r_{k}}\right\|_{2}^{2}=\left\|e_{t}^{k}\right\|_{2}^{2}+\left\|x_{r_{k}}\right\|_{2}^{2}+2 x_{r_{k}} e_{t}^{k} \\
& =-\left\|e_{t}^{k}\right\|_{2}^{2}+\frac{1}{2}\left\|x_{r_{k}}\right\|_{2}^{2}+\left\|\sqrt{2} e_{t}^{k}+\frac{1}{\sqrt{2}} x_{r_{k}}\right\|_{2}^{2} \geq-\left\|e_{t}^{k}\right\|_{2}^{2}+\frac{1}{2}\left\|x_{r_{k}}\right\|_{2}^{2} .
\end{aligned}
$$

Applying equation (28) into equation (27), we obtain

$$
\begin{aligned}
\dot{V} & \leq-\beta^{2}\left\|x_{t}\right\|_{2}^{2}-\left(1-\beta^{2}\right)\left(-\left\|e_{t}^{k}\right\|_{2}^{2}+\frac{1}{2}\left\|x_{r_{k}}\right\|_{2}^{2}\right)+\left(e_{t}^{k}\right)^{T} Q e_{t}^{k}-x_{r_{k}}^{T} Q x_{r_{k}}+\gamma^{2}\left\|w_{t}\right\|_{2}^{2} \\
& =-\beta^{2}\left\|x_{t}\right\|_{2}^{2}+\gamma^{2}\left\|w_{t}\right\|_{2}^{2}+\left(e_{t}^{k}\right)^{T} M e_{t}^{k}-x_{r_{k}}^{T} N x_{r_{k}},
\end{aligned}
$$

where $M$ and $N$ are defined by equation (5) and (6), respectively.

Proof: [Proof of Lemma 3.2] Notice that

$$
\begin{aligned}
& \dot{x}_{t}=A x_{t}-B_{1} B_{1}^{T} P x_{r_{k-1}}+B_{2} w_{t}, \quad \forall t \in\left[r_{k}, f_{k}\right), \quad \text { and } \\
& \dot{x}_{t}=A x_{t}-B_{1} B_{1}^{T} P x_{r_{k}}+B_{2} w_{t}, \quad \forall t \in\left[f_{k}, f_{k+1}\right)\left[r_{k}, f_{k}\right) .
\end{aligned}
$$

We first consider $z_{t}^{k}$ over the time interval $\left[r_{k}, f_{k}\right)$. Let $\Phi_{1}=\left\{t \in\left[r_{k}, f_{k}\right):\left\|z_{t}^{k}\right\|_{2}=0\right\}$. The time derivative of $\left\|z_{t}^{k}\right\|_{2}$ for $t \in\left[r_{k}, f_{k}\right) \backslash \Phi_{1}$ satisfies

$$
\begin{aligned}
\frac{d}{d t}\left\|z_{t}^{k}\right\|_{2} & \leq\left\|\sqrt{M} \dot{e}_{t}^{k}\right\|_{2}=\left\|\sqrt{M} \dot{x}_{t}\right\|_{2}=\left\|\sqrt{M}\left(A x_{t}-B_{1} B_{1}^{T} P x_{r_{k-1}}+B_{2} w_{t}\right)\right\|_{2} \\
& \leq \alpha\left\|z_{t}^{k}\right\|_{2}+\mu_{1}\left(x_{r_{k}}, x_{r_{k-1}}\right)+\left\|\sqrt{M} B_{2}\right\|\left\|w_{t}\right\|_{2},
\end{aligned}
$$

where the righthand sided derivative is used when $t=r_{k}$.

Using standard comparison principle on the preceding equation over the interval $t \in\left[r_{k}, f_{k}\right)$ with the initial condition $\left\|z_{r_{k}}^{k}\right\|_{2}=0$, we have

$$
\left\|z_{t}^{k}\right\|_{2} \leq \frac{\mu_{1}\left(x_{r_{k}}, x_{r_{k-1}}\right)}{\alpha}\left(e^{\alpha\left(t-r_{k}\right)}-1\right)+\int_{r_{k}}^{t} e^{\alpha(t-s)}\left\|\sqrt{M} B_{2}\right\|\left\|w_{s}\right\|_{2} d s
$$


for all $t \in\left[r_{k}, f_{k}\right)$ because $\left\|z_{t}^{k}\right\|_{2}=0$ for all $t \in \Phi_{1}$.

Let $\Phi_{2}=\left\{t \in\left[f_{k}, f_{k+1}\right):\left\|z_{t}^{k}\right\|_{2}=0\right\}$. The time derivative of $\left\|z_{t}^{k}\right\|_{2}$ for $t \in\left[f_{k}, f_{k+1}\right) \backslash \Phi_{2}$ satisfies

$$
\frac{d}{d t}\left\|z_{t}^{k}\right\|_{2} \leq \alpha\left\|z_{t}^{k}\right\|_{2}+\mu_{0}\left(x_{r_{k}}\right)+\left\|\sqrt{M} B_{2}\right\|\left\|w_{t}\right\|_{2},
$$

where the righthand sided derivative is used when $t=f_{k}$.

Using standard comparison principle on the preceding equation over the interval $t \in\left[f_{k}, f_{k+1}\right)$ with the initial condition

$$
\left\|z_{f_{k}}^{k}\right\|_{2} \leq \frac{\mu_{1}\left(x_{r_{k}}, x_{r_{k-1}}\right)}{\alpha}\left(e^{\alpha D_{k}}-1\right)+\int_{r_{k}}^{f_{k}} e^{\alpha\left(f_{k}-s\right)}\left\|\sqrt{M} B_{2}\right\|\left\|w_{s}\right\|_{2} d s
$$

obtained from equation (29), we have

$$
\begin{aligned}
\left\|z_{t}^{k}\right\|_{2} \leq & e^{\alpha\left(t-f_{k}\right)} \frac{\mu_{1}\left(x_{r_{k}}, x_{r_{k-1}}\right)}{\alpha}\left(e^{\alpha D_{k}}-1\right)+e^{\alpha\left(t-f_{k}\right)} \int_{r_{k}}^{f_{k}} e^{\alpha\left(f_{k}-s\right)}\left\|\sqrt{M} B_{2}\right\|\left\|w_{s}\right\|_{2} d s \\
& +\frac{\mu_{0}\left(x_{r_{k}}\right)}{\alpha}\left(e^{\alpha\left(t-f_{k}\right)}-1\right)+\int_{f_{k}}^{t} e^{\alpha(t-s)}\left\|\sqrt{M} B_{2}\right\|\left\|w_{s}\right\|_{2} d s
\end{aligned}
$$

holds for all $t \in\left[f_{k}, f_{k+1}\right)$ since $\left\|z_{t}^{k}\right\|_{2}=0$ for all $t \in \Phi_{2}$.

Proof: [Proof of Theorem 3.3] Since the hypotheses in lemma 3.2 hold, equation (30) holds. By squaring both sides of the inequality in equation (30), we obtain

$$
\begin{aligned}
\left\|z_{t}^{k}\right\|_{2}^{2} \leq & 4\left[e^{\alpha\left(t-f_{k}\right)} \frac{\mu_{1}\left(x_{r_{k}}, x_{r_{k-1}}\right)}{\alpha}\left(e^{\alpha D_{k}}-1\right)\right]^{2}+4\left[\frac{\mu_{0}\left(x_{r_{k}}\right)}{\alpha}\left(e^{\alpha\left(t-f_{k}\right)}-1\right)\right]^{2} \\
& +4 e^{2 \alpha\left(t-f_{k}\right)} J^{2}\left[w ; r_{k}, f_{k}\right]+4 J^{2}\left[w ; f_{k}, t\right]
\end{aligned}
$$

holds for all $t \in\left[f_{k}, f_{k+1}\right)$.

By lemma 3.1, we know

$$
\dot{V} \leq-\beta^{2}\left\|x_{t}\right\|_{2}^{2}+\gamma^{2}\left\|w_{t}\right\|_{2}^{2}+\left\|z_{t}^{k}\right\|_{2}^{2}-x_{r_{k}}^{T} N x_{r_{k}}
$$

holds for all $t \in\left[f_{k}, f_{k+1}\right)$ with $V(x)=x^{T} P x$. Applying equation (31) into the preceding inequality implies that

$$
\begin{aligned}
\dot{V} \leq & -\beta^{2}\left\|x_{t}\right\|_{2}^{2}+\gamma^{2}\left\|w_{t}\right\|_{2}^{2}-x_{r_{k}}^{T} N x_{r_{k}}+4 e^{2 \alpha\left(t-f_{k}\right)} J^{2}\left[w ; r_{k}, f_{k}\right]+4 J^{2}\left[w ; f_{k}, t\right] \\
& +4\left[e^{\alpha\left(t-f_{k}\right)} \frac{\mu_{1}\left(x_{r_{k}}, x_{r_{k-1}}\right)}{\alpha}\left(e^{\alpha D_{k}}-1\right)\right]^{2}+4\left[\frac{\mu_{0}\left(x_{r_{k}}\right)}{\alpha}\left(e^{\alpha\left(t-f_{k}\right)}-1\right)\right]^{2}
\end{aligned}
$$


holds for all $t \in\left[f_{k}, f_{k+1}\right)$. Integrating both sides of the preceding inequality on $t$ over $\left[f_{k}, f_{k+1}\right)$ yields

$$
\begin{aligned}
\int_{f_{k}}^{f_{k+1}} \dot{V} d t \leq & -\int_{f_{k}}^{f_{k+1}} \beta^{2}\left\|x_{t}\right\|_{2}^{2} d t+\int_{f_{k}}^{f_{k+1}} \gamma^{2}\left\|w_{t}\right\|_{2}^{2} d t-\int_{f_{k}}^{f_{k+1}} x_{r_{k}}^{T} N x_{r_{k}} d t \\
& +\int_{f_{k}}^{f_{k+1}} 4 e^{2 \alpha\left(t-f_{k}\right)} J^{2}\left[w ; r_{k}, f_{k}\right] d t+\int_{f_{k}}^{f_{k+1}} 4 J^{2}\left[w ; f_{k}, t\right] d t \\
& +\int_{f_{k}}^{f_{k+1}} 4\left[e^{\alpha\left(t-f_{k}\right)} \frac{\mu_{1}\left(x_{r_{k}}, x_{r_{k-1}}\right)}{\alpha}\left(e^{\alpha D_{k}}-1\right)\right]^{2} d t \\
& +\int_{f_{k}}^{f_{k+1}} 4\left[\frac{\mu_{0}\left(x_{r_{k}}\right)}{\alpha}\left(e^{\alpha\left(t-f_{k}\right)}-1\right)\right]^{2} d t
\end{aligned}
$$

Let us look at the last two terms in the preceding equation. Notice that

$$
\begin{aligned}
& \int_{f_{k}}^{f_{k+1}} 4\left[e^{\alpha\left(t-f_{k}\right)} \frac{\mu_{1}\left(x_{r_{k}}, x_{r_{k-1}}\right)}{\alpha}\left(e^{\alpha D_{k}}-1\right)\right]^{2} d t+\int_{f_{k}}^{f_{k+1}} 4\left[\frac{\mu_{0}\left(x_{r_{k}}\right)}{\alpha}\left(e^{\alpha\left(t-f_{k}\right)}-1\right)\right]^{2} d t \\
= & \frac{2 \mu_{1}^{2}\left(x_{r_{k}}, x_{r_{k-1}}\right)}{\alpha^{3}}\left(e^{\alpha D_{k}}-1\right)^{2}\left(e^{2 \alpha\left(f_{k+1}-f_{k}\right)}-1\right)+\frac{2 \mu_{0}^{2}\left(x_{r_{k}}\right)}{\alpha^{3}}\left(e^{2 \alpha\left(f_{k+1}-f_{k}\right)}-1\right) \\
& -\frac{8 \mu_{0}^{2}\left(x_{r_{k}}\right)}{\alpha^{3}}\left(e^{\alpha\left(f_{k+1}-f_{k}\right)}-1\right)+\frac{4 \mu_{0}^{2}\left(x_{r_{k}}\right)}{\alpha^{2}}\left(f_{k+1}-f_{k}\right) \\
\leq & \left(x_{r_{k}}^{T} N x_{r_{k}}+\delta_{k}\right)\left(f_{k+1}-f_{k}\right)=\int_{f_{k}}^{f_{k+1}} x_{r_{k}}^{T} N x_{r_{k}} d t+\delta_{k}\left(f_{k+1}-f_{k}\right)
\end{aligned}
$$

holds, where the inequality is obtained using equation (18).

Applying equation (34), together with equations (16) and (17), into equation (33) yields

$$
\begin{aligned}
\int_{f_{k}}^{f_{k+1}} \dot{V} d t \leq & -\int_{f_{k}}^{f_{k+1}} \beta^{2}\left\|x_{t}\right\|_{2}^{2} d t+\int_{f_{k}}^{f_{k+1}} \gamma^{2}\left\|w_{t}\right\|_{2}^{2} d t+\delta_{k}\left(\tau_{1}+\tau_{2}\right) \\
& +\int_{f_{k}}^{f_{k+1}} 4 e^{2 \alpha\left(t-f_{k}\right)} J^{2}\left[w ; r_{k}, f_{k}\right] d t+\int_{f_{k}}^{f_{k+1}} 4 J^{2}\left[w ; f_{k}, t\right] d t
\end{aligned}
$$

Let us now look at the fourth item in the right side of the inequality in equation (35). Using Cauchy-Schwarz inequality, we have

$$
\begin{aligned}
J^{2}\left[w ; r_{k}, f_{k}\right] & =\left(\int_{r_{k}}^{f_{k}} e^{\alpha\left(f_{k}-s\right)}\left\|\sqrt{M} B_{2}\right\|\left\|w_{s}\right\|_{2} d s\right)^{2} \\
& \leq\left(\int_{r_{k}}^{f_{k}} e^{\alpha\left(f_{k}-s\right)} d s\right)\left(\int_{r_{k}}^{f_{k}} e^{\alpha\left(f_{k}-s\right)}\left\|\sqrt{M} B_{2}\right\|^{2}\left\|w_{s}\right\|_{2}^{2} d s\right)
\end{aligned}
$$


for all $t \in\left[f_{k}, f_{k+1}\right)$. Therefore,

$$
\begin{aligned}
& \int_{f_{k}}^{f_{k+1}} 4 e^{2 \alpha\left(t-f_{k}\right)} J^{2}\left[w ; r_{k}, f_{k}\right] d t \\
\leq & \left(\int_{f_{k}}^{f_{k+1}} 4 e^{2 \alpha\left(t-f_{k}\right)} d t\right)\left(\int_{r_{k}}^{f_{k}} e^{2 \alpha\left(f_{k}-s\right)} d s\right)\left(\int_{r_{k}}^{f_{k}}\left\|\sqrt{M} B_{2}\right\|^{2}\left\|w_{s}\right\|_{2}^{2} d s\right) \\
= & \frac{2}{\alpha}\left(e^{2 \alpha\left(f_{k+1}-f_{k}\right)}-1\right) \frac{1}{2 \alpha}\left(e^{2 \alpha\left(f_{k}-r_{k}\right)}-1\right)\left\|\sqrt{M} B_{2}\right\|^{2} \int_{r_{k}}^{f_{k}}\left\|w_{s}\right\|_{2}^{2} d s \\
\leq & \frac{1}{\alpha^{2}}\left(e^{2 \alpha\left(\tau_{1}+\tau_{2}\right)}-1\right)\left(e^{2 \alpha \tau_{2}}-1\right)\left\|\sqrt{M} B_{2}\right\|^{2} \int_{f_{k-1}}^{f_{k}}\left\|w_{s}\right\|_{2}^{2} d s
\end{aligned}
$$

holds, where the last inequality is obtained using equations (16) and (17).

Following the similar analysis, we obtain an upper bound on the fifth item in the right side of the inequality in equation (35):

$$
\int_{f_{k}}^{f_{k+1}} 4 J^{2}\left[w ; f_{k}, t\right] d t \leq \frac{4\left\|\sqrt{M} B_{2}\right\|^{2}}{\alpha^{2}}\left(e^{\alpha\left(\tau_{1}+\tau_{2}\right)}-1\right)^{2} \int_{f_{k}}^{f_{k+1}}\left\|w_{s}\right\|_{2}^{2} d s .
$$

Applying equations (38) and (39) into (35), we obtain

$$
\begin{aligned}
\int_{f_{k}}^{f_{k+1}} \dot{V} d t \leq & -\beta^{2} \int_{f_{k}}^{f_{k+1}}\left\|x_{t}\right\|_{2}^{2} d t+\gamma^{2} \int_{f_{k}}^{f_{k+1}}\left\|w_{t}\right\|_{2}^{2} d t+\delta_{k}\left(\tau_{1}+\tau_{2}\right) \\
& +\frac{\left(e^{2 \alpha\left(\tau_{1}+\tau_{2}\right)}-1\right)\left(e^{2 \alpha \tau_{2}}-1\right)\left\|\sqrt{M} B_{2}\right\|^{2}}{\alpha^{2}} \int_{f_{k-1}}^{f_{k}}\left\|w_{s}\right\|_{2}^{2} d s \\
& +\frac{4\left\|\sqrt{M} B_{2}\right\|^{2}}{\alpha^{2}}\left(e^{\alpha\left(\tau_{1}+\tau_{2}\right)}-1\right)^{2} \int_{f_{k}}^{f_{k+1}}\left\|w_{s}\right\|_{2}^{2} d s
\end{aligned}
$$

Summarizing $k$ in the inequality above from 0 to $\infty$ yields

$$
\int_{0}^{\infty} \dot{V} d t \leq-\beta^{2} \int_{0}^{\infty}\left\|x_{t}\right\|_{2}^{2} d t+\left(\tau_{1}+\tau_{2}\right) \sum_{k=0}^{\infty} \delta_{k}+\eta^{2} \int_{0}^{\infty}\left\|w_{s}\right\|_{2}^{2} d s
$$

where $\eta$ is defined in equation (20).

Since $\sum_{k=0}^{\infty} \delta_{k} \leq \infty$, the inequality above is sufficient to show the sampled-data system is finite-gain $\mathcal{L}_{2}$ stable from $w$ to $x$ with an induced gain less than $\eta$.

Proof: [Proof of Theorem 3.6] We will show the self-triggering scheme defined in equations (23) and (25) satisfies equation (15) - (18) in Theorem 3.3.

It is obvious that equations (23) and (25) imply the satisfaction of the inequalities in equations (16) and (17), respectively. By the assumption, $M$ defined in equation (5) has full rank. As a 
result, $N$ defined in equation (6) also has full rank and $M \geq N>0$. Therefore, by the definitions of $L_{2}$ and $L_{3}$ in equations (24) and (26), we have

$$
\begin{aligned}
& L_{2}\left(x_{r_{k}}\right) \geq \frac{1}{\alpha} \ln \left(1+\frac{\alpha \sqrt{\lambda_{\min }(N)}}{\sqrt{8 \lambda_{\max }\left(A_{\mathrm{cl}}^{T} M A_{\mathrm{cl}}\right)}}\right)>0, \\
& L_{3}\left(x_{r_{k+1}}, x_{r_{k}} ; \delta_{k+1}\right) \geq 0 .
\end{aligned}
$$

Therefore, by equations (23) and (25), $r_{k} \leq f_{k} \leq r_{k+1}$ holds for all $k \in \mathbb{N}$.

We now show the satisfaction of equation (18). By equation (23) and (25), we have

$$
\begin{aligned}
& f_{k+1}-f_{k} \leq \min \left\{\tau_{1}+\tau_{2}, L_{2}\left(x_{r_{k}}\right)\right\}, \\
& D_{k} \leq L_{3}\left(x_{r_{k}}, x_{r_{k-1}} ; \delta_{k}\right) .
\end{aligned}
$$

With equations (41) and (42), we have

$$
\begin{aligned}
& 4 e^{2 \alpha\left(t-f_{k}\right)} \frac{\mu_{1}^{2}\left(x_{r_{k}}, x_{r_{k-1}}\right)}{\alpha^{2}}\left(e^{\alpha D_{k}}-1\right)^{2} \leq 4 e^{2 \alpha\left(f_{k+1}-f_{k}\right)} \frac{\mu_{1}^{2}\left(x_{r_{k}}, x_{r_{k-1}}\right)}{\alpha^{2}}\left(e^{\alpha D_{k}}-1\right)^{2} \\
\leq & 4 e^{2 \alpha \min \left\{\tau_{1}+\tau_{2}, L_{2}\left(x_{r_{k}}\right)\right\}} \frac{\mu_{1}^{2}\left(x_{r_{k}}, x_{r_{k-1}}\right)}{\alpha^{2}}\left(e^{\alpha D_{k}}-1\right)^{2} \leq \frac{1}{2} x_{r_{k}}^{T} N x_{r_{k}}+\delta_{k}
\end{aligned}
$$

holds for all $t \in\left[f_{k}, f_{k+1}\right)$.

Also, equation (41) implies

$$
\frac{4 \mu_{0}^{2}\left(x_{r_{k}}\right)}{\alpha^{2}}\left(e^{\alpha\left(t-f_{k}\right)}-1\right)^{2} \leq \frac{4 \mu_{0}^{2}\left(x_{r_{k}}\right)}{\alpha^{2}}\left(e^{\alpha L_{2}\left(x_{r_{k}}\right)}-1\right)^{2} \leq \frac{1}{2} x_{r_{k}}^{T} N x_{r_{k}}
$$

holds for all $t \in\left[f_{k}, f_{k+1}\right)$.

Combining equation (43) and (44) yields

$$
4 e^{2 \alpha\left(t-f_{k}\right)} \frac{\mu_{1}^{2}\left(x_{r_{k}}, x_{r_{k-1}}\right)}{\alpha^{2}}\left(e^{\alpha D_{k}}-1\right)^{2}+\frac{4 \mu_{0}^{2}\left(x_{r_{k}}\right)}{\alpha^{2}}\left(e^{\alpha\left(t-f_{k}\right)}-1\right)^{2} \leq x_{r_{k}}^{T} N x_{r_{k}}+\delta_{k}
$$

for all $t \in\left[f_{k}, f_{k+1}\right)$.

Integrating both sides of the preceding inequality on $t$ over $\left[f_{k}, f_{k+1}\right)$ implies the satisfaction of equation (18). Since the hypotheses in Theorem 3.3 are satisfied, we can conclude that the sampled-data system is finite-gain $\mathcal{L}_{2}$ stable from $w$ to $x$ with an induced gain less than a positive constant $\eta$. 


\section{REFERENCES}

[1] K. Astrom and B. Wittenmark, Computer-Controlled Systems: theory and design, 3rd ed. Prentice-Hall, 1997.

[2] Y. Zheng, D. Owens, and S. Billings, "Fast sampling and stability of nonlinear sampled-data systems: Part 2. sampling rate estimations," IMA Journal of Mathematical Control and Information, vol. 7, pp. 13-33, 1990.

[3] D. Nesic, A. Teel, and E. Sontag, "Formulas relating $\mathcal{K} \mathcal{L}$ stability estimates of discrete-time and sampled-data nonlinear systems," Systems and Control Letters, vol. 38, pp. 49-60, 1999.

[4] L. Zaccarian, A. Teel, and D. Nesic, "On finite gain $\mathcal{L}_{p}$ stability of nonlinear sampled-data systems," System and Control Letters, vol. 49, pp. 201-212, 2003.

[5] G. Walsh, H. Ye, and L. Bushnell, "Stability analysis of networked control systems," IEEE Transactions on Control Systems Technology, vol. 10, no. 3, pp. 438-446, 2002.

[6] D. Nesic and A. Teel, "Input-output stability properties of networked control systems," IEEE Transactions on Automatic Control, vol. 49, pp. 1650-1667, 2004.

[7] D. Carnevale, A. R. Teel, and D. Nesic, "Further results on stability of networked control systems: a lyapunov approach," IEEE Transactions on Automatic Control, vol. 52, pp. 892-897, 2007.

[8] K. Arzen, "A simple event-based PID controller," in Proceedings of the 14th IFAC World Congress, 1999.

[9] D. Hristu-Varsakelis and P. Kumar, "Interrupt-based feedback control over a shared communication medium," in Proceedings of IEEE Conference on Decision and Control, 2002.

[10] K. Astrom and B. Bernhardsson, "Comparison of riemann and lebesgue sampling for first order stochastic systems," in Proceedings of IEEE Conference on Decision and Control, 1999.

[11] P. Tabuada and X. Wang, "Preliminary results on state-triggered scheduling of stabilizing control tasks," in Proceedings of IEEE Conference on Decision and Control, 2006.

[12] P. Tabuada, "Event-Triggered Real-Time Scheduling of Stabilizing Control Tasks," Automatic Control, IEEE Transactions on, vol. 52, no. 9, pp. 1680-1685, 2007.

[13] X. Wang and M. Lemmon, "Self-triggered feedback control systems with finite-gain $\mathcal{L}_{2}$ stability," to appear in IEEE Transactions on Automatic Control.

[14] M. Velasco, P. Marti, and J. Fuertes, "The self triggered task model for real-time control systems," in Work-in-Progress Session of the 24th IEEE Real-Time Systems Symposium (RTSSO3), 2003.

[15] M. Lemmon, T. Chantem, X. Hu, and M. Zyskowski, "On self-triggered full information h-infinity controllers," in Hybrid Systems: computation and control, 2007.

[16] A. Anta and P. Tabuada, "Self-triggered stabilization of homogeneous control systems," in American Control Conference, 2008. 